\title{
Artificial Intelligence in dentistry: Concepts, Applications and Research Challenges
}

\author{
Achsha Babu ${ }^{1}$, J. Andrew Onesimu ${ }^{2}$, and K. Martin Sagayam ${ }^{3}$ \\ ${ }^{1}$ Department of Biotechnolgy, Karunya Institute of Technology and Sciences, Coimbatore, India. \\ ${ }^{2}$ Department of Computer Science and Engineering, Karunya Institute of Technology and Sciences, Coimbatore, India. \\ ${ }^{3}$ Department of Electronics and Communication Engineering, Karunya Institute of Technology and Sciences, Coimbatore, India.
}

\begin{abstract}
Artificial Intelligence (AI) has the ability to process huge datasets, disclose human essence computationally, and perform like humans as technology advances. Because of the necessity for precise diagnosis and improved patient care, AI technology has greatly influenced the healthcare industry. In the domains of dentistry and medicine, artificial intelligence has yet to come a long way. As a result, dentists must be aware of the potential implications for a profitable clinical practise in the future. In this paper, we present the current applications of AI in dentistry. The different types of AI techniques are introduced and summarized. The state-of-the-art literature is studied analysed. A comparative analysis on the different AI techniques in dentistry is presented. Further, the research challenges in the field of dentistry and future directions are also provided.
\end{abstract}

\section{Introduction}

Artificial intelligence (AI) has emerged throughout the world to mimic human intelligence and tackle certain challenges [1]. AI can assist in the development of algorithms that can learn from information provided and make predictions. Machine learning creates algorithms based on data. One of the first type of AI algorithms to be developed was neural networks (NNs) [2]. Deep learning neural networks are structures with numerous levels and enormous layers, whereas shallow learning neural networks are simple network structures with only a few layers [3].

Convolutional neural networks (CNNs) are mostly used to analyse vast and complicated images. In 1955, a mathematician named John McCarthy coined the term artificial intelligence, earning the title "Father of Artificial Intelligence" [4]. In 1978, Richard Bellman a mathematician, defined artificial intelligence as the automation of processes related to human cognitive capacities such as learning, decision making, and problem solving [5].

It is essential to be aware of key elements of artificial intelligence systems in society today, as indicated in Figure 1.

- Artificial intelligence is a machine's ability to express its own intelligence by solving problems based on data.

- Machine learning uses algorithms to anticipate outcomes from a set of data. The goal is to make it easier for machines without human intervention to learn from data and solve problems.

\footnotetext{
* Corresponding author: andrewj@karunya.edu
}

- Neural networks compute signals using artificial neurons which operates similar to human brain.

- Deep learning with multiple computational layers builds a neural network that automatically recognises patterns in order to improve feature detection [6].

- Data science is the process of analyzing data and extracting useful information from it [7].

- Big data provides users with accurate information by assessing a vast set of data that has been continually growing for years at the right time [8].

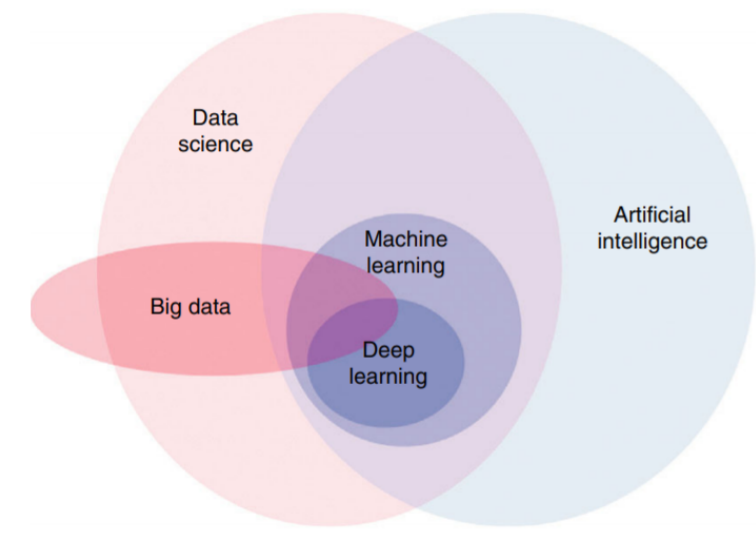

Fig. 1. Key elements of artificial intelligent systems [9]

Algorithms accomplish tasks by learning patterns of data in machine learning. Machine learning needs adjusting elements such as neural network neurons and layers, population size, mutation rate, and crossing over rate in genetic algorithms, depending on the underlying 
approach. The artificial neuron is one of the components of ANN. A network capable of handling certain tasks like image classification is generated by creating artificial neurons and linking the layers using multiple mathematical procedures (e.g., During RCT, a radiographic image of a damaged tooth is used to locate canals). ANN with a complicated multilayer structure is Deep Learning (DL). This system has a more complicated layering system and a larger number of associated neurons, allowing it to see simple features such as lines, edges, corners, and macro patterns in a hierarchical framework [10].

AI and its implications have been viewed as both a benefit and a curse during the last seven decades. Several times this technology fails to match expectations. However, previous decade on the other hand, is recognised as the decade of major advances in the field of artificial intelligence. Its convincing that people couldn't tell whether AI is penned by a human or a machine. The face recognition system is another advancement. Finally, AI-based technologies transform from a scourge to a blessing, influencing all aspects of society, including healthcare and politics [11]. AI may now be utilised to solve real-world problems thanks to rapid technology breakthroughs [12]. In medical field, the deep learning algorithms is providing a high level of accuracy in diagnostics. There isn't much progress on specific dental problems. Hence, a deep insight is required for dental study. The current state and performance of AI in dentistry applications is yet to be well recorded and analyzed. Therefore, the current review explores how AI and machine learning algorithms are used in dental diseases and diagnostics.

Artificial Intelligence technique have a lot of potential for detecting and diagnosing oral cavity abnormalities that go unnoticed by the human eye, thus they're making their way into orthodontic care [13]. Machine learning algorithms, deep learning techniques like artificial neural networks (ANN), Convolutional neural networks $(\mathrm{CNN})$, genetic algorithms (GA), and fuzzy logic are some of the AI approaches being used in dentistry.

\section{Machine Learning in Image Processing}

Data is pre-processed and manually labelled before being sent to the ML algorithm for training. Before the mature model is finalized, selected ML algorithms are employed for data learning, followed by model optimization and model evaluation. The steps involved in medical image processing are picture capture, preprocessing, analysis, and identification of pattern. Preprocessed images are collected from X-rays and MRI scan images [14]. Then, for image processing use CNN models. The retrieved feature information must now be fed into the modelling process. On the basis of the feedback from the results, fall-back processes such as hyperparameter adjustment and model adjustment are carried out [15]. The fundamental machine learning technique is shown in Fig. 2.

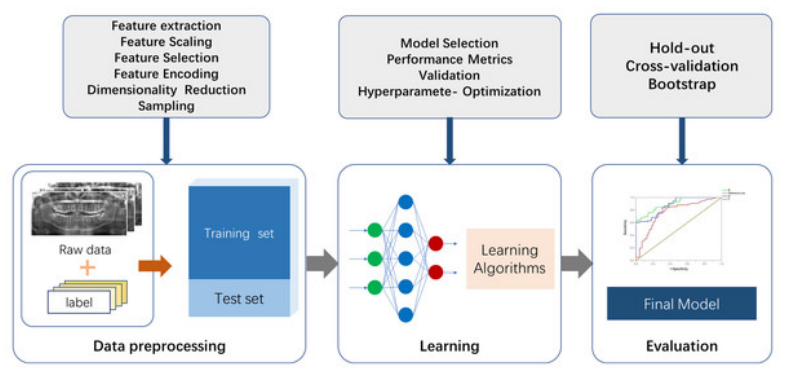

Fig. 2. Machine learning technique to get final model using image processing [15]

\section{Artificial Neural Network}

Minsky and Dean Edmunds created the stochastic neural analogue reinforcement calculator in 1951, which is widely regarded as the first neural network [1]. The main benefit of these systems is that they can solve problems that are too complex. Kim et al. utilized an artificial neural network to create a model that can predict toothache based on the relationship between toothache and daily tooth brushing frequency, time, use of dental floss, toothbrush replacement pattern, scaling, and other parameters such as nutrition and activity [16]. This successful study contributed in the construction of a highly accurate toothache prediction model. The criteria to meet in reducing toothaches are proper eating habits, oral cleanliness, and stress reduction. Another study employed Bayesian network analysis to discover correlations between numerous parameters affecting the diagnostic and treatment result of maxillary canines that were damaged. All of patient's treatment data, as well as their records were obtained for this study in order to find correlations between these factors. Some researchers developed an artificial neural network model to anticipate the need for extraction during orthodontic therapy [17]. Data mining research of a large number of patient's restorative data found that variances in tooth restoration material are major determinants in influencing the lifespan. Its responsibility lies in capacity to discover causal links and comparisons that are already present in existing data. In a study, Käkilehto et al. analyzed survival curves of new restorative materials on occlusal surface [18]. Data mining analysis was used for observation, and the dental professional has to only gather and tabulate the data. Speight et al. in 1995 used ANN for risk assessment of oral cancer to study tooth infection [19]. Devito along with his colleagues studied AI based model for diagnosing the proximal dental caries in tooth decay [20]. In 2010, Xie et al. used an ANN model to determine if extractions are required prior for dental malocclusion in orthodontic treatment [21]. In tooth treatment, Saghiri et al. used the ANN approach to locate the minor apical foramen (AF) [22]. Jung and Kim studied the diagnosis of orthodontic extraction in tooth using ANN [23]. In 2017 Nino-Sandoval et al. predicted the mandibular morphology of anatomical landmarks [24]. Using ANN model, the skeletal 
diagnostic of anatomical landmarks was studied by $\mathrm{Yu}$ and co-workers [25]. Patil et al. observed ANN for gender determination of mandible part [26]. Javed et al. used ANN model to predict post-Streptococcus mutans favours in the selection of caries excavation strategy, which eventually leads in meliorate caries-free cavity using iOS App is shown in Fig. 3. [27].

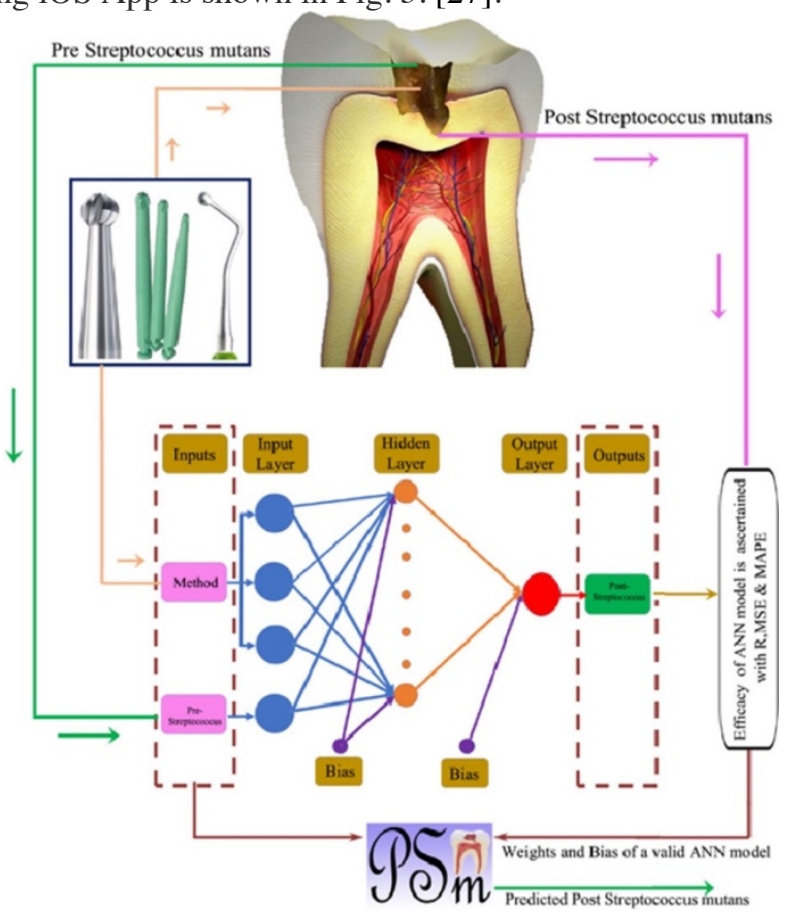

Fig. 3. ANN model to predict post-Streptococcus mutans to treat caries excavation [27]

As a result, artificial intelligence is primarily focused on developing powerful systems to assist dental professionals in making decisions as well as to assist patients in understanding their conditions and prognosis. Finally, dentists perform the real examination, diagnosis, and treatment, with AI serving only to aid the dental expert.

\section{Genetic Algorithms}

During the recent decade, genetic algorithms have also acquired popularity as implementations for complicated issues. They are swarm intelligence methods based on the natural selection concept of survival of the fittest. To find a better solution to the problem, it employs techniques such as mutation, inheritance, selection, and crossover. The most significant advantage of genetic algorithms over traditional approaches is that they are based on problem solving rather than the analytical relations notion. Despite being a strong optimization tool, it is straightforward to use because it is based on basic criteria. Lodygowski and his colleagues to eliminate the problem of physical fracture and offer long-term strength to the implant used genetic algorithms to optimise the dental implant system [28].

Another study by Li $\mathrm{H}$ et al. combined a genetic algorithm with a back propagation neural network to improve tooth colour matching, which is one of the most difficult aspects of prosthetic dentistry[29]. A new study described a genetic algorithm-based strategy for detecting dental caries early and preventing decay severity [30]. In computer assisted design, a genetic algorithm is also used to repair missing tooth parts by optimising it. This research found that utilising genetic algorithms to manage the reconstructed surface and preserve overall smoothness, there is a lot of room for geometric modelling and optimization strategies.

\section{Fuzzy Logic}

We may encounter situations in our daily lives where we are unable to determine if a statement is true or incorrect. Fuzzy logic provides very useful reasoning for the fuzzy situation at that time. Lotfi Zadeh created the fuzzy logic. Fuzzy logic is a machine learning methodology that is both versatile and simple to use. The four major components of the Fuzzy Logic system are as follows: 1) Rule Basse 2) Fuzzification 3) Inference Engine 4) Defuzzification. The goal of fuzzy logic is to mimic human reasoning abilities that function with ambiguous concepts. To aid dental professionals in making decisions, Mago et al. created a fuzzy logic based expert system that allows imprecise values of dental signsymptoms linked with mobile teeth [31]. Ambara and co-workers devised a fuzzy logic-based expert system that is both time and cost effective, and can be conducted without the use of any equipment reducing the patient's stress [32]. Hererra and associates employed a fuzzy logic-based approach to anticipate the change in colour after bleaching based on the original chrome values of the teeth [33]. A set of rules was carried out to correlate pre-bleaching hues.

\section{Convolutional Neural Network}

In research, CNNs are being used for dental imaging diagnosis. Later, more rigorous, replicable, and comparable procedures should be used to demonstrate their utility, safety, and generalizability. Using a convolutional neural network $(\mathrm{CNN})$, Eun and his colleagues observed directed tooth localisation for periapical dental X-ray images [34]. Imangaliyev and his co-workers in 2016 worked on CNN model for classification of dental plaque images using 427 datasets [35]. In 2017, Aubreville et al. for diagnosing oral squamous cell carcinoma (OSCC) in oral cavity used AI-based approach [36]. In 2017, De Tobel and his colleagues for estimating age using panoramic radiographs to stage lower third molar growth identified an automated technique [37]. Lee et al. worked on CNN model for diagnosis and prediction of periodontally compromised teeth [38]. Yauney et al. for automated health assessments and cross-correlations of oralsystemic health used AI-based CNN model [39]. Detection and diagnosis of dental caries using CNN in 2018 was examined. Also, treatment outcome analysis in teeth was done by Patcas et al. in 2018 [40]. Zhang et $a l$. for teeth recognition used label tree with cascade network structure [41]. 
Table 1. AI Based State-of-the art Literature in Dentistry

\begin{tabular}{|c|c|c|c|c|c|}
\hline Author & Year & Technique & $\begin{array}{l}\text { Dataset } \\
\text { Images } \\
\text { (Count) }\end{array}$ & Description & Applications \\
\hline Speight et al. [19] & 1995 & ANN & 1662 & Oral cancer risk assessment & Oral infection \\
\hline Devito et al. [20] & 2008 & ANN & 160 & $\begin{array}{l}\text { A model based on artificial intelligence for } \\
\text { identifying proximal dental caries }\end{array}$ & Tooth decay \\
\hline Xie et al. [21] & 2010 & ANN & 200 & $\begin{array}{l}\text { An artificial intelligence approach for determining } \\
\text { whether extractions are required before orthodontic } \\
\text { treatment. }\end{array}$ & $\begin{array}{l}\text { Tooth } \\
\text { malocclusion }\end{array}$ \\
\hline Saghiri et al. [22] & 2012 & ANN & 50 & $\begin{array}{l}\text { ANN system to locate the minor apical foramen } \\
\text { (AF) }\end{array}$ & Tooth \\
\hline Jung \& Kim [23] & 2016 & ANN & 156 & Orthodontic extraction diagnosis & $\begin{array}{l}\text { Tooth } \\
\text { malocclusion }\end{array}$ \\
\hline $\begin{array}{l}\text { Imangaliyev et al. } \\
\text { [35] }\end{array}$ & 2016 & $\mathrm{CNN}$ & 427 & $\begin{array}{l}\text { Classification of dental plaque samples using deep } \\
\text { learning }\end{array}$ & Dental plaque \\
\hline Eun et al. [34] & 2016 & $\mathrm{CNN}$ & 500 & $\begin{array}{l}\text { Periapical dental X-ray imaging for oriented tooth } \\
\text { localization }\end{array}$ & Tooth \\
\hline $\begin{array}{l}\text { Aubreville et al. } \\
\text { [36] }\end{array}$ & 2017 & $\mathrm{CNN}$ & 7894 & $\begin{array}{l}\text { Oral squamous cell carcinoma (OSCC) diagnosis } \\
\text { using AI systems }\end{array}$ & Oral cavity \\
\hline $\begin{array}{l}\text { De Tobel et al. } \\
\text { [37] }\end{array}$ & 2017 & $\mathrm{CNN}$ & 200 & $\begin{array}{l}\text { An automated method for predicting age using } \\
\text { panoramic radiographs to grade lower third molar } \\
\text { growth. }\end{array}$ & Tooth \\
\hline Johari et al. [54] & 2017 & PNN & 240 & $\begin{array}{l}\text { VRFs in undamaged and endodontically treated } \\
\text { teeth are diagnosed using a probabilistic neural } \\
\text { network. }\end{array}$ & Tooth \\
\hline $\begin{array}{l}\text { Thanathorn } \\
\text { wong[53] }\end{array}$ & 2018 & $\begin{array}{l}\text { Bayesian } \\
\text { networks }\end{array}$ & 1000 & Clinical decision support in tooth using AI & Tooth \\
\hline Patcas et al. [40] & 2018 & $\mathrm{CNN}$ & 2164 & Analysis of tooth treatment outcomes & Tooth \\
\hline Zhang et al. [41] & 2018 & $\mathrm{CNN}$ & 100 & $\begin{array}{l}\text { A label tree with a cascade network structure is used } \\
\text { to recognise teeth. }\end{array}$ & Tooth \\
\hline Lee et al. [38] & 2018 & $\mathrm{CNN}$ & 600 & Dental caries detection and diagnosis & Tooth (caries) \\
\hline Feres et al. [55] & 2018 & SVM & 3915 & $\begin{array}{l}\text { Periodontitis classification as either aggressive or } \\
\text { chronic. }\end{array}$ & Periodontitis \\
\hline $\begin{array}{l}\text { Casalegno et al. } \\
{[42]}\end{array}$ & 2019 & $\mathrm{CNN}$ & 217 & $\begin{array}{l}\text { In Near-Infrared Transillumination (TI) images, an } \\
\text { AI-based model for recognising and localising } \\
\text { dental lesions has been developed. }\end{array}$ & Dental caries \\
\hline Krois et al. [47] & 2019 & $\mathrm{CNN}$ & 2001 & Periodontal bone loss detection system & Periodontium \\
\hline Chen et al. [61] & 2019 & $\mathrm{CNN}$ & 250 & $\begin{array}{l}\text { A toolkit based on CNN for recognising and } \\
\text { counting teeth. }\end{array}$ & Tooth \\
\hline Ekert et al. [44] & 2019 & $\mathrm{CNN}$ & 2001 & $\begin{array}{l}\text { An artificial intelligence method for detecting apical } \\
\text { lesions has been developed (ALs) }\end{array}$ & Tooth \\
\hline Fukuda et al. [45] & 2019 & $\mathrm{CNN}$ & 60 & AI to detect vertical root fractures. & Tooth \\
\hline Hiraiwa et al. [46] & 2019 & $\mathrm{CNN}$ & 760 & $\begin{array}{l}\text { AI to classify the root morphologies of mandibular } \\
\text { first molars. }\end{array}$ & Tooth \\
\hline Tuzoff et al. [48] & 2019 & $\mathrm{CNN}$ & 222 & $\begin{array}{l}\text { Automatic tooth detection and counting using } \\
\text { artificial intelligence }\end{array}$ & Tooth \\
\hline $\begin{array}{l}\text { Vinayahalingam } \\
\text { et al. [51] }\end{array}$ & 2019 & $\mathrm{CNN}$ & 81 & $\begin{array}{l}\text { On OPGs, the inferior alveolar nerve (IAN) to lower } \\
\text { third molars roots was detected. }\end{array}$ & Tooth \\
\hline Yu et al. [25] & 2020 & ANN & 5890 & Skeletal diagnostic system & $\begin{array}{l}\text { Anatomical } \\
\text { landmarks }\end{array}$ \\
\hline Patil et al. [26] & 2020 & ANN & 509 & ANN for determining gender & Mandible \\
\hline $\begin{array}{l}\text { Schwendicke et } \\
\text { al. [50] }\end{array}$ & 2020 & $\mathrm{CNN}$ & 226 & $\begin{array}{l}\text { In near-infrared light transillumination (NILT) } \\
\text { images, } C N N \text { can detect caries lesions. }\end{array}$ & Tooth decay \\
\hline Leite et al. [52] & 2020 & $\mathrm{CNN}$ & 851 & $\begin{array}{l}\text { Predicted dental condition based on reports taken } \\
\text { from a panoramic radiograph }\end{array}$ & Tooth \\
\hline
\end{tabular}


Table 2. Comparative analysis of various AI techniques

\begin{tabular}{|c|c|c|c|}
\hline Algorithms & Benefits & Drawbacks & Uses \\
\hline ANN & $\begin{array}{l}\text { - Robustness } \\
\text { - Have a high level of fault tolerance } \\
\text { - Has the ability to solve tough } \\
\text { problems } \\
\text { - They have memory that is } \\
\text { dispersed. }\end{array}$ & $\begin{array}{l}\text { - Hardware is required. } \\
\text { - Network behaviour } \\
\text { unaddressed } \\
\text { - Selection of proper network } \\
\text { structure }\end{array}$ & $\begin{array}{l}\text { It is employed in the } \\
\text { development of a toothache } \\
\text { prediction model for the } \\
\text { treatment of dental infection } \\
\text { and decay. }\end{array}$ \\
\hline BAYESIAN & $\begin{array}{l}\text { - They outperform other techniques } \\
\text { of learning. } \\
\text { - Possibility of making probabilistic } \\
\text { predictions }\end{array}$ & $\begin{array}{l}\text { - Many possibilities must be } \\
\text { understood at the start. } \\
\text { - It's possible that it'll become } \\
\text { computationally intractable. }\end{array}$ & $\begin{array}{l}\text { It is used in clinical decision- } \\
\text { making systems. }\end{array}$ \\
\hline $\mathrm{CNN}$ & $\begin{array}{l}\text { - Simple to comprehend and put into } \\
\text { practice } \\
\text { - It predicts images with accuracy and } \\
\text { is computationally efficient without } \\
\text { the need for human intervention. } \\
\text { - Weight sharing }\end{array}$ & $\begin{array}{l}\text { - They don't retain the object's } \\
\text { position or orientation. } \\
\text { - It is necessary to collect a } \\
\text { large amount of training data. } \\
\text { - Inability to be spatially } \\
\text { invariant to the received data }\end{array}$ & $\begin{array}{l}\text { It is used in treatment of } \\
\text { periodontics and dental caries. }\end{array}$ \\
\hline PNN & $\begin{array}{l}\text { - PNNs surpass multilayer perceptron } \\
\text { networks in terms of speed and } \\
\text { accuracy. } \\
\text { - Outliers aren't as noticeable in PNN } \\
\text { networks. }\end{array}$ & $\begin{array}{l}\text { - More memory is required to } \\
\text { store the model. }\end{array}$ & $\begin{array}{l}\text { Used for root canal therapy for } \\
\text { the treatment of damaged } \\
\text { teeth. }\end{array}$ \\
\hline SVM & $\begin{array}{l}\text { - In high-dimensional spaces, SVM is } \\
\text { more efficient. } \\
\text { - SVM requires a small amount of } \\
\text { memory. }\end{array}$ & $\begin{array}{l}\text { - For big data sets, the SVM } \\
\text { algorithm is ineffective. } \\
\text { - The SVM will underperform } \\
\text { if the number of features for } \\
\text { each data point exceeds the } \\
\text { number of training data } \\
\text { samples. }\end{array}$ & $\begin{array}{l}\text { Based on supervised training, } \\
\text { it is used to classify and } \\
\text { identify dental images as } \\
\text { either normal or pathological } \\
\text { flaws. }\end{array}$ \\
\hline
\end{tabular}

In Near-Infrared Transillumination, Casalegno et al. used CNN approach to detect and localise dental lesions images [42]. For recognising and counting the teeth, Chen and his colleagues employed a CNN-based toolkit [43]. Ekert studied AI system for detecting apical lesions (ALs) in teeth [44]. For detection of vertical root fracture in tooth Fukuda and his associates performed CNN modelling [45]. Hiraiwa et al. classified root morphologies of mandibular first molars using CNN approach [46]. Krois et al. using CNN developed a system for detecting periodontal bone loss [47]. In 2019, CNN for automatic teeth detection and numbering was examined by Tuzoff et al [48]. Lee et al. used CNN on classification of specific features of osteoporosis for face [49]. Schwendicke et al. in Near-Infrared light transillumination detected caries lesions [50]. Vinayahalingam using 81 datasets in total, on OPGs detected the Inferior Alveolar Nerve (IAN) to lower third molars roots using CNN networks [51]. Leite and his colleagues collaborated to combine two neural networks for recognising and segmenting teeth using CNN to predict dental condition based on reports taken from a panoramic radiograph is explained in Figure 4 [52].Equations should be centred and should be numbered with the number on the right-hand side.

Thanathorn using Bayesian networks studied AI clinical decision support in tooth [53]. Johari et al. diagnosed (VRFs) in intact and the teeth under endodontic treatment using Probabilistic Neural Network (PNN) [54]. Feres et al.in 2018 used Support Vector Machine classification (SVM) for differentiation between aggressive and chronic periodontitis [55]. AI technology that has been used over the years as described in literatures are summarized in Table 1.

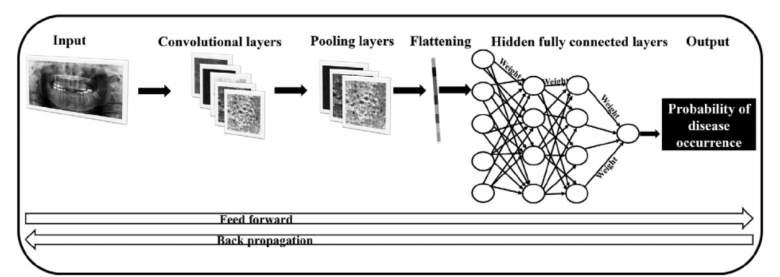

Fig. 4. CNN model to forecast the patient's dental condition from a panoramic radiograph [52]

Also, Intraoral scanners and cameras are used to diagnose and arrange treatment using radiographs and photos. This eliminates the need for patient impressions as well as multiple laboratory stages, and the results are typically far more precise than human perception. Algorithms and statistical analysis can be used to anticipate tooth movement and treatment outcome [21]. Table 2 lists the benefits, drawbacks, and uses of various AI approaches.

\section{Research Challenges and Future Directions}

Despite their potential, AI technologies are yet to have a significant impact in medical practise. Convolutional neural networks (CNNs) in dentistry are presently being developed [56]. Tackling certain issues will help to make dental AI technology better and facilitate their uptake in clinical care. For interpretation dentistry uses imaging data which is frequently collected over a period 
of time. Medical and dental data are not readily available and accessible as other data. Data is frequently kept away in siloed, personalised, and only loosely interoperable systems. Each patient's data is complicated and sensitive, and there are few choices for interpreting or validating it. Data obtained from electronic medical records, has a low level of variable completeness, with data missing systematically rather than at random. Because data is intrinsically skewed, sampling frequently leads to prejudice [57].Data processing, measurement, and validation are frequently insufficiently reproducible and robust [58]. It's still a mystery how datasets were chosen, curated, and preprocessed. Data is frequently used for both training and testing, leading to a phenomenon known as data snooping bias [57] [58]. The single data offered by most current dental AI applications will only partially inform the required and complex clinical decision-making [59]. Furthermore, there are still concerns about accountability and transparency.

As we see advancements in science and technology, Artificial Intelligence has risen to prominence as a huge phenomenon. It will continue to be a technological pioneer for the foreseeable future, based on its current growth rate. In order to focus on individualised treatment with patient-centred outcomes, dental research should develop the link between oral and general health in the future. In this perspective, dental research must have an impact on society as a deliverable, not merely research that produces scientific papers but research that actually changes clinical protocols.

In case of digital dentistry, this necessitates a realistic approach to managing expectations and providing openness for all. There are various obstacles in collecting, storing, and analysing digitised medical patient data. Aside from the technical requirements of handling large volumes of data while adhering to recognized standards, an ethical and meaningful policy must assure the security of patient data for the best possible outcome in terms of safety. Oncologists may find that radiomics can help them forecast the metastatic potential of tumours and the expression of oncogenes, as well as assess therapy response. Computational models could leverage data from imaging biobanks to simulate illness onset and progression in the future [60].

The technological advances combines the utilization of robotic support in dentistry. Also in today's scenario, "augmented intelligence" has been adopted a little too soon. Nonetheless, the advantages of digital applications will complement human skills and abilities in order to provide patients with better and more costeffective healthcare. Big data-based augmented intelligence can help to reduce the number of misdiagnoses and provide more meaningful insights rapidly, correctly, and effortlessly

\section{Conclusion}

Artificial intelligence models have the potential to be a useful tool for diagnosing caries and tooth dysfunctionality. The dental applications of AI models, on the other hand, are still in the works. Future research could include developing an automated method to anticipate periodontitis as a decision-making aid, particularly in diabetic patients. Watson by IBM has been used to assist doctors in making therapeutic choices [61]. The clinical accuracy of AI in dentistry must be tested using a variety of imaging sources before AI can play a larger role in diagnostic judgments, due to the difficulty of standardising dental radiography. Furthermore, contemporary AI algorithms are opaque, making it difficult for humans to recognise or modify diagnostic criteria [62]. As a result, a visualisation and modification tool for deep learning networks can be easily understood and altered by humans to improve AI's reliability if needed. Artificial intelligence can help make considerable advances in providing better health care to patients, but it will never be able to replace human knowledge, skills, or judgement.

\section{References}

1. W. J. Park and J.-B. Park, "History and application of artificial neural networks in dentistry," European Journal of Dentistry, vol. 12, no. 4, pp. 594-601, 2018, doi: 10.4103/ejd.ejd_325_18.

2. M. Mupparapu, C.-W. Wu, and Y.-C. Chen, "Artificial intelligence, machine learning, neural networks, and deep learning: Futuristic concepts for new dental diagnosis," Quintessence International (Berlin, Germany: 1985), vol. 49, no. 9, pp. 687-688, 2018, doi: 10.3290/j.qi.a41107.

3. J. R. Burt et al., "Deep learning beyond cats and dogs: recent advances in diagnosing breast cancer with deep neural networks," The British Journal of Radiology, vol. 91, no. 1089, Sep. 2018, doi: 10.1259/bjr.20170545.

4. V. Rajaraman, "JohnMcCarthy — Father of artificial intelligence,” Resonance, vol. 19, no. 3, pp. 198-207, Mar. 2014, doi: 10.1007/s12045014-0027-9.

5. R. Bellman, An introduction to artificial intelligence: can computers think? San Francisco: Boyd \& Fraser Pub. Co., 1978.

6. S. B. Khanagar et al., "Developments, application, and performance of artificial intelligence in dentistry - A systematic review," Journal of Dental Sciences, Jun. 2020, doi: 10.1016/j.jds.2020.06.019.

7. M. L. Brodie, "What Is Data Science?," Applied Data Science, pp. 101-130, 2019, doi: 10.1007/978-3-030-11821-1_8.

8. Y. Riahi and S. Riahi, "Big Data and Big Data Analytics: concepts, types and technologies," International Journal of Research and Engineering, 
vol. 5, no. 9, pp. 524-528, Nov. 2018, doi: 10.21276/ijre.2018.5.9.5.

9. P. I. Dorado-Díaz, J. Sampedro-Gómez, V. Vicente-Palacios, and P. L. Sánchez, "Applications of Artificial Intelligence in Cardiology. The Future is Already Here," Revista Española de Cardiología (English Edition), vol. 72, no. 12, pp. 1065-1075, Dec. 2019, doi: 10.1016/j.rec.2019.05.014.

10. S. A. Kareem, P. Pozos-Parra, and N. Wilson, “An application of belief merging for the diagnosis of oral cancer," Applied Soft Computing, vol. 61, pp. 1105-1112, Dec. 2017, doi: 10.1016/j.asoc.2017.01.055.

11. A. Yaji, S. Prasad, and A. Pai, "ACTA SCIENTIFIC DENTAL SCIENCES (ISSN: 25814893) Artificial Intelligence in DentoMaxillofacial Radiology," https://actascientific.com/ASDS/pdf/ASDS-030423.pdf.

12. J.R. Rabuñal, J. Dorado. Artificial neural networks in real-life applications. IGI Global: Hershey (2005), pp. 166-346.

13. A. Kalappanavar, S. Sneha, and R. G. Annigeri, "Artificial intelligence: A dentist's perspective," Journal of Medicine, Radiology, Pathology and Surgery, vol. 5, no. 2, pp. 2-4, 2018, doi: 10.15713/ins.jmrps.123.

14. S. Asgari Taghanaki, K. Abhishek, J. P. Cohen, J. Cohen-Adad, and G. Hamarneh, "Deep semantic segmentation of natural and medical images: a review," Artificial Intelligence Review, vol. 54, no. 1, pp. 137-178, Jan. 2021, doi: 10.1007/s10462-020-09854-1.

15. R. Ren, H. Luo, C. Su, Y. Yao, and W. Liao, "Machine learning in dental, oral and craniofacial imaging: a review of recent progress," PeerJ, vol. 9, p. e11451, May 2021, doi: 10.7717/peerj.11451.

16. E. Y. Kim, K. O. Lim, and H. S. Rhee, "Predictive modeling of dental pain using neural network," Studies in Health Technology and Informatics, vol. 146, pp. 745-746, 2009, Available: https://pubmed.ncbi.nlm.nih.gov/19592958/.

17. M. Nieri, A. Crescini, R. Rotundo, T. Baccetti, P. Cortellini, and G. P. Pini Prato, "Factors affecting the clinical approach to impacted maxillary canines: A Bayesian network analysis," American Journal of Orthodontics and Dentofacial Orthopedics: Official Publication of the American Association of Orthodontists, Its Constituent Societies, and the American Board of Orthodontics, vol. 137, no. 6, pp. 755-762, Jun. 2010, doi: 10.1016/j.ajodo.2008.08.028.

18. T. Käkilehto, S. Salo, and M. Larmas, "Data mining of clinical oral health documents for analysis of the longevity of different restorative materials in Finland," International Journal of Medical Informatics, vol. 78, no. 12, pp. e68-74, Dec. 2009, doi: 10.1016/j.ijmedinf.2009.04.004.
19. P. M. Speight, A. E. Elliott, J. A. Jullien, M. C. Downer, and J. M. Zakzrewska, "The use of artificial intelligence to identify people at risk of oral cancer and precancer," British Dental Journal, vol. 179 , no. 10 , pp. 382-387, Nov. 1995, doi: 10.1038/sj.bdj.4808932.

20. K. L. Devito, F. de Souza Barbosa, and W. N. Felippe Filho, "An artificial multilayer perceptron neural network for diagnosis of proximal dental caries," Oral Surgery, Oral Medicine, Oral Pathology, Oral Radiology, and Endodontics, vol. 106, no. 6, pp. 879-884, Dec. 2008, doi: 10.1016/j.tripleo.2008.03.002.

21. X. Xie, L. Wang, and A. Wang, “Artificial Neural Network Modeling for Deciding if Extactions Are Necessary Prior to Orthodontic Treatment," The Angle Orthodontist, vol. 80, no. 2, pp. 262-266, Mar. 2010, doi: 10.2319/111608-588.1.

22. M. A. Saghiri et al., "A new approach for locating the minor apical foramen using an artificial neural network," International Endodontic Journal, vol. 45, no. 3, pp. 257-265, Mar. 2012, doi: 10.1111/j.1365-2591.2011.01970.x.

23. S.-K. Jung and T.-W. Kim, "New approach for the diagnosis of extractions with neural network machine learning," American Journal of Orthodontics and Dentofacial Orthopedics: Official Publication of the American Association of Orthodontists, Its Constituent Societies, and the American Board of Orthodontics, vol. 149, no. 1, pp. 127-133, Jan. 2016, doi: 10.1016/j.ajodo.2015.07.030.

24. T. C. Niño-Sandoval, S. V. Guevara Pérez, F. A. González, R. A. Jaque, and C. Infante-Contreras, "Use of automated learning techniques for predicting mandibular morphology in skeletal class I, II and III," Forensic Science International, vol. 281, pp. 187.e1-187.e7, Dec. 2017, doi: 10.1016/j.forsciint.2017.10.004.

25. H. J. Yu, S. R. Cho, M. J. Kim, W. H. Kim, J. W. Kim, and J. Choi, "Automated Skeletal Classification with Lateral Cephalometry Based on Artificial Intelligence," Journal of Dental Research, vol. 99, no. 3, pp. 249-256, Mar. 2020, doi: 10.1177/0022034520901715.

26. V. Patil et al., “Artificial neural network for gender determination using mandibular morphometric parameters: A comparative retrospective study," Cogent Engineering, vol. 7, no. 1 , p. 1723783 , Jan. 2020 , doi: 10.1080/23311916.2020.1723783.

27. S. Javed, M. Zakirulla, R. U. Baig, S. M. Asif, and A. B. Meer, "Development of artificial neural network model for prediction of poststreptococcus mutans in dental caries," Computer Methods and Programs in Biomedicine, vol. 186, p. 105198, Apr. 2020, doi: 10.1016/j.cmpb.2019.105198.

28. T. Łodygowski, K. Szajek, and M. Wierszycki, "Optimization of dental implant using genetic algorithm," undefined, 2009. 
https://www.semanticscholar.org/paper/Optimizati on-of-dental-implant-using-genetic-

\%C5\%81odygowski

Szajek/4d80c478c1b71803aa95085724ab250785f $\underline{1 \mathrm{dc} 25}$.

29. H. Li, L. Lai, L. Chen, C. Lu, and Q. Cai, "The Prediction in Computer Color Matching of Dentistry Based on GA+BP Neural Network," Computational and Mathematical Methods in Medicine, Mar. 22, 2015. https://www.hindawi.com/journals/cmmm/2015/8 $\underline{16719 /}$.

30. P. Tripathi, C. Malathy and M. Prabhakaran, "Genetic algorithms-based approach for dental caries detection using back propagation neural network," Int J Recent Technol Eng. 2019;8 2277 3878.

31. V. K. Mago, A. Mago, P. Sharma, and J. Mago, "Fuzzy logic based expert system for the treatment of mobile tooth," Advances in Experimental Medicine and Biology, vol. 696, pp. 607-614, 2011, doi: 10.1007/978-1-4419-7046-6_62.

32. B. Ambara, D. Putra, and D. Rusjayanthi, "Fuzzy Expert System of Dental and Oral Disease with Certainty Factor," International Journal of Computer Science Issues, vol. 14, no. 3, pp. 2230, May 2017, doi: 10.20943/01201703.2230.

33. L. J. Herrera et al., "Prediction of color change after tooth bleaching using fuzzy logic for Vita Classical shades identification," Applied Optics, vol. 49, no. 3, pp. 422-429, Jan. 2010, doi: 10.1364/AO.49.000422.

34. H. Eun and C. Kim, "Oriented tooth localization for periapical dental X-ray images via convolutional neural network," 2016 Asia-Pacific Signal and Information Processing Association Annual Summit and Conference (APSIPA), 2016, doi: 10.1109/APSIPA.2016.7820720.

35. S. Imangaliyev, M. H. Veen, C. Volgenant, B. Keijser, W. Crielaard, and E. Levin, "Deep Learning for Classification of Dental Plaque Images," Semantic Scholar, 2016.

https://www.semanticscholar.org/paper/DeepLearning-for-Classification-of-Dental-PlaqueImangaliyev-

Veen/ad48783363e3d4ed0c913256afca672a31f2fa $\underline{77}$.

36. M. Aubreville et al., "Automatic Classification of Cancerous Tissue in Laser endomicroscopy Images of the Oral Cavity using Deep Learning," Scientific Reports, vol. 7, no. 1, p. 11979, Sep. 2017, doi: 10.1038/s41598-017-12320-8.J.

37. [37]De Tobel, P. Radesh, D. Vandermeulen, and P. W. Thevissen, "An automated technique to stage lower third molar development on panoramic radiographs for age estimation: a pilot study," The Journal of Forensic Odonto-Stomatology, vol. 35, no. 2 , pp. $42-54$, Dec. 2017 , Available: https://pubmed.ncbi.nlm.nih.gov/29384736/.
38. J.-H. Lee, D.-H. Kim, S.-N. Jeong, and S.-H. Choi, "Detection and diagnosis of dental caries using a deep learning-based convolutional neural network algorithm," Journal of Dentistry, vol. 77, pp. 106111, Oct. 2018, doi: 10.1016/j.jdent.2018.07.015.

39. G. Yauney, A. Rana, L. C. Wong, P. Javia, A. Muftu, and P. Shah, "Automated Process Incorporating Machine Learning Segmentation and Correlation of Oral Diseases with Systemic Health," arXiv:1810.10664 [cs, q-bio, stat], Oct. 2018, Accessed: Jun. 14, 2021. [Online]. Available: https://arxiv.org/abs/1810.10664.

40. R. Patcas, D. A. J. Bernini, A. Volokitin, E. Agustsson, R. Rothe, and R. Timofte, "Applying artificial intelligence to assess the impact of orthognathic treatment on facial attractiveness and estimated age," International Journal of Oral and Maxillofacial Surgery, vol. 48, no. 1, pp. 77-83, Jan. 2019, doi: 10.1016/j.ijom.2018.07.010.W.

41. Zhang, J. Li, Z.-B. Li, and Z. Li, "Predicting postoperative facial swelling following impacted mandibular third molars extraction by using artificial neural networks evaluation," Scientific Reports, vol. 8, no. 1, Aug. 2018, doi: 10.1038/s41598-018-29934-1.

42. F. Casalegno et al., "Caries Detection with NearInfrared Transillumination Using Deep Learning," Journal of Dental Research, vol. 98, no. 11, pp. 1227-1233, Oct. 2019, doi: $10.1177 / 0022034519871884$.

43. H. Chen et al., "A deep learning approach to automatic teeth detection and numbering based on object detection in dental periapical films," Scientific Reports, vol. 9, no. 1, p. 3840, Mar. 2019, doi: 10.1038/s41598-019-40414-y.

44. T. Ekert et al., "Deep Learning for the Radiographic Detection of Apical Lesions," Journal of Endodontics, vol. 45, no. 7, pp. 917922.e5, Jul. 2019, doi: 10.1016/j.joen.2019.03.016.

45. M. Fukuda et al., "Evaluation of an artificial intelligence system for detecting vertical root fracture on panoramic radiography," Oral Radiology, Sep. 2019, doi: 10.1007/s11282-01900409-x.

46. T. Hiraiwa et al., "A deep-learning artificial intelligence system for assessment of root morphology of the mandibular first molar on panoramic radiography," Dentomaxillo facial Radiology, vol. 48, no. 3, p. 20180218, Mar. 2019, doi: 10.1259/dmfr.20180218.

47. J. Krois et al., "Deep Learning for the Radiographic Detection of Periodontal Bone Loss," Scientific Reports, vol. 9, no. 1, Jun. 2019, doi: 10.1038/s41598-019-44839-3.

48. D. V. Tuzoff et al., "Tooth detection and numbering in panoramic radiographs using convolutional neural networks," Dento Maxillo Facial Radiology, vol. 48, no. 4, p. 20180051, May 2019, doi: 10.1259/dmfr.20180051. 
49. K.-S. Lee, S.-K. Jung, J.-J. Ryu, S.-W. Shin, and J. Choi, "Evaluation of Transfer Learning with Deep Convolutional Neural Networks for Screening Osteoporosis in Dental Panoramic Radiographs," Journal of Clinical Medicine, vol. 9, no. 2, Feb. 2020, doi:10.3390/jcm9020392.

50. F. Schwendicke, K. Elhennawy, S. Paris, P. Friebertshäuser, and J. Krois, "Deep learning for caries lesion detection in near-infrared light transillumination images: A pilot study," Journal of Dentistry, vol. 92, p. 103260, Jan. 2020, doi: 10.1016/j.jdent.2019.103260.

51. S. Vinayahalingam, T. Xi, S. Bergé, T. Maal, and G. de Jong, "Automated detection of third molars and mandibular nerve by deep learning," Scientific Reports, vol. 9, no. 1, p. 9007, Jun. 2019, doi: 10.1038/s41598-019-45487-3.

52. A. F. Leite, K. de F. Vasconcelos, H. Willems, and R. Jacobs, "Radiomics and Machine Learning in Oral Healthcare," Proteomics. Clinical Applications, vol. 14, no. 3, p. e1900040, May 2020, doi: 10.1002/prca.201900040.

53. B. Thanathornwong, "Bayesian-Based Decision Support System for Assessing the Needs for Orthodontic Treatment," Healthcare Informatics Research, vol. 24, no. 1, pp. 22-28, Jan. 2018, doi: 10.4258/hir.2018.24.1.22.

54. M. Johari, F. Esmaeili, A. Andalib, S. Garjani, and H. Saberkari, "Detection of vertical root fractures in intact and endodontically treated premolar teeth by designing a probabilistic neural network: an ex vivo study," Dentomaxillofacial Radiology, vol. 46, no. 2, doi: 10.1259/dmfr.20160107.

55. M. Feres, Y. Louzoun, S. Haber, M. Faveri, L. C. Figueiredo, and L. Levin, "Support vector machine-based differentiation between aggressive and chronic periodontitis using microbial profiles," International Dental Journal, vol. 68, no. 1, pp. 39-46, Feb. 2018, doi: 10.1111/idj.12326.

56. F. Schwendicke, T. Golla, M. Dreher, and J. Krois, "Convolutional neural networks for dental image diagnostics: A scoping review," Journal of Dentistry, vol. 91, p. 103226, Dec. 2019, doi: 10.1016/j.jdent.2019.103226.

57. M. A. Gianfrancesco, S. Tamang, J. Yazdany, and G. Schmajuk, "Potential Biases in Machine Learning Algorithms Using Electronic Health Record Data," JAMA Internal Medicine, vol. 178, no. 11 , p. 1544, Nov. 2018, doi: 10.1001/jamainternmed.2018.3763.

58. J. R. England and P. M. Cheng, "Artificial Intelligence for Medical Image Analysis: A Guide for Authors and Reviewers," American Journal of Roentgenology, vol. 212, no. 3, pp. 513-519, Mar. 2019, doi: 10.2214/ajr.18.20490.

59. T. M. Maddox, J. S. Rumsfeld, and P. R. O. Payne, "Questions for Artificial Intelligence in Health Care," JAMA, vol. 321, no. 1, p. 31, Jan. 2019, doi: 10.1001/jama.2018.18932.
60. A. Hosny, C. Parmar, J. Quackenbush, L. H. Schwartz, and H. J. W. L. Aerts, "Artificial intelligence in radiology," Nature Reviews Cancer, vol. 18, no. 8, pp. 500-510, May 2018, doi: 10.1038/s41568-018-0016-5.

61. Chen, J. Elenee Argentinis, and G. Weber, "IBM Watson: How Cognitive Computing Can Be Applied to Big Data Challenges in Life Sciences Research," Clinical Therapeutics, vol. 38, no. 4, pp. 688-701, Apr. 2016, doi: 10.1016/j.clinthera.2015.12.001.

62. F. A. Anifowose, "Artificial Intelligence Application in Reservoir Characterization and Modeling: Whitening the Black Box," onepetro.org, Mar. 14, 2011. https://onepetro.org/SPEYPTS/proceedingsabstract/11YPTS/All-11YPTS/SPE-155413$\mathrm{MS} / 150902$ 